Article

\title{
Risk Assessment for Distribution Systems Using an Improved PEM-Based Method Considering Wind and Photovoltaic Power Distribution
}

\author{
Qingwu Gong, Jiazhi Lei *, Hui Qiao and Jingjing Qiu \\ School of Electrical Engineering, Wuhan University, Wuhan 430072, China; qwgong@whu.edu.cn (Q.G.); \\ qiaohui@whu.edu.cn (H.Q.); 15072305723@163.com (J.Q.) \\ * Correspondence: leijiazhi@126.com; Tel.: +86-27-68772281 \\ Academic Editors: Gwo-Hshiung Tzeng and Kao-Yi Shen \\ Received: 31 December 2016; Accepted: 16 March 2017; Published: 24 March 2017
}

\begin{abstract}
The intermittency and variability of permeated distributed generators (DGs) could cause many critical security and economy risks to distribution systems. This paper applied a certain mathematical distribution to imitate the output variability and uncertainty of DGs. Then, four risk indices-EENS (expected energy not supplied), PLC (probability of load curtailment), EFLC (expected frequency of load curtailment), and SI (severity index)—were established to reflect the system risk level of the distribution system. For the certain mathematical distribution of the DGs' output power, an improved PEM (point estimate method)-based method was proposed to calculate these four system risk indices. In this improved PEM-based method, an enumeration method was used to list the states of distribution systems, and an improved PEM was developed to deal with the uncertainties of DGs, and the value of load curtailment in distribution systems was calculated by an optimal power flow algorithm. Finally, the effectiveness and advantages of this proposed PEM-based method for distribution system assessment were verified by testing a modified IEEE 30-bus system. Simulation results have shown that this proposed PEM-based method has a high computational accuracy and highly reduced computational costs compared with other risk assessment methods and is very effective for risk assessments.
\end{abstract}

Keywords: distributed generators; risk assessment; distribution systems; improved PEM-based method; optimal power flow algorithm

\section{Introduction}

The extensive penetration of renewable-type distributed generators (DGs) (e.g., wind and PV) in distribution networks could bring many benefits to the grid, as they are alternative to conventional generators [1,2]. However, the randomness of these DGs could cause some critical risks to security and economy aspects of distribution systems, such as power quality and stability, fault level, and the value of load curtailment, which impose challenges when planning distribution systems [3,4]. Therefore, it is becoming increasingly important to assess the risks associated with the variability and uncertainty of DGs.

In the past decade, many researches concentrated on how to assess the impacts of random DGs on distribution systems. Many risk indices have been established in [5-8]. These papers aimed to establish the risk indices by using the product of probability and severity, but ignored many significant aspects such as the value of load curtailment. Many other risk indices have also been presented. In [9], three risk indices-including LOLP (loss of load probability), EENS (expected energy not supplied), and ECOST (expected customer interruption cost)—were used for reliability and price risk assessment. In [10,11], the indices of SAIFI (system average interruption frequency index), SAIDI 
(system average interruption duration index), and EENS were used to assess the reliability of an active distribution network. In [12], EENS, ENLC (expected number of load curtailment), and ADLC (average duration of load curtailment) were used for power system reliability assessment. In [13-15], EENS, PLC (probability of load curtailment), EFLC (expected frequency of load curtailment), and SI (severity index) were presented as risk indices to assess the risk level of distribution system. In [9-15], more considerations were concentrated on the operation risk level. Needless to say, EENS, PLC, EFLC, and SI are the most important risk indices that reflect the high penetration of DGs in distribution system.

The output uncertainties of DGs can be dealt with by many uncertainty modeling methods including probabilistic methods and other uncertainty methods [16]. Among the other uncertainty methods, IGDT (information gap decision theory), robust optimization, and a hybrid possibilistic-probabilistic approach were respectively introduced in [17-19]. However, the power output of wind or photovoltaic (PV) generating units is generally subject to a certain mathematical distribution $[20,21]$. Namely, the randomness of wind and PV can be imitated by some mathematical formulas. Therefore, these other uncertainty methods mentioned are not applicable for the risk assessment of a distribution system with the penetration of probabilistic DGs. For probabilistic DGs, probabilistic methods-including the Monte Carlo method, scenario-based decision-making method, and point estimate method—can be suitably applied. However, the Monte Carlo method (which was introduced in [22,23]) and the scenario-based decision-making method (introduced in [24]) are all computationally harder. Compared to the other probabilistic methods, the point estimate method (PEM), described in [25-27], has highly reduced computational costs and is extremely applicable for the probabilistic uncertainties of DGs. However, the accuracy of PEM is very low. Therefore, the traditional PEM should be improved for application.

In this study, in order to reasonably assess the risks of distribution systems with the penetration of DGs, a risk assessment method for distribution systems using an improved PEM-based method-considering wind and photovoltaic power distribution-was proposed. Four risk indices (EENS, PLC, EFLC, and SI) were applied to reflect the system risk level of distribution systems. Then, for the certain mathematical distribution of the DGs' output power, an improved PEM-based method was proposed to calculate these four system risk indices. In this improved PEM-based method, an enumeration method was used to list the states of the distribution system; an improved PEM was proposed to deal with the uncertainties of DGs in the distribution system. Finally, the effectiveness and advantage of this proposed PEM-based method for power system assessment were verified by testing a modified IEEE 30-bus system, which showed that this proposed PEM-based method is very effective for risk assessments in distribution systems and has a high computational accuracy and largely reduced computational costs compared with other risk assessment methods. Simulation results also demonstrate that total generation capacity, type, location, dispersion, and capacity proportion of DGs have great influences on the system risk indices.

\section{Distribution of Wind and Photovoltaic DGs}

In distribution systems, the output of DGs contributes to whether the load can be supplied when malfunction occurs. Therefore, the output randomness of DGs should be imitated by mathematical formulas. In this paper, wind generators and photovoltaic generators are only considered as DGs.

\subsection{Output Uncertainty of Wind Generators}

Large amounts of research in the past decades have demonstrated that wind speed $v$ is the main stochastic factor that determines the output power of wind generators. Generally, a Weibull distribution can be used to imitate the stochastic wind speed $v$ [6]. The probability density function (PDF) for wind speed $v$ is described as (1):

$$
f_{v}(v)=\frac{k}{c}\left(\frac{v}{c}\right) \exp \left[-\left(\frac{v}{c}\right)^{k}\right]
$$


where $k$ and $c$ are, respectively, the shape parameter and the scale parameter of the wind speed distribution. According to the historical data of wind speed $v$, these two indices can be estimated.

Based on the PDF of wind speed $v$, the output of a wind turbine generator can be acquired [28]:

$$
P_{w}=\left\{\begin{array}{cc}
0, & v<v_{\text {in }} \\
P_{N} \frac{v-v_{\text {in }}}{v_{N}-v_{\text {in }},} & v_{\text {in }} \leq v<v_{N} \\
P_{N}, & v_{N} \leq v<v_{\text {out }} \\
0, & v \geq v_{\text {out }}
\end{array}\right.
$$

where $P_{w}$ is the active output power, $P_{N}$ is the rated output power of wind turbine generator, $v_{N}$ is rated wind speed, $v_{\text {in }}$ is cut-in wind speed, and $v_{\text {out }}$ is cut-out wind speed.

\subsection{Output Uncertainty of Photovoltaic Generators}

In most occasions, illumination intensity is thought to be the major factor that affects the active output of photovoltaic generators. Because of cloud cover and other insolation-reducing phenomena, the illumination intensity $I$ can also be represented by a random variable. In general, illumination intensity $I$ approximately follows a beta distribution with shape indexes $\alpha$ and $\beta$ [29]:

$$
f(I)=\frac{\Gamma(\alpha+\beta)}{\Gamma(\alpha) \Gamma(\beta)} \cdot\left(\frac{I}{I_{\max }}\right)^{\alpha-1} \cdot\left(1-\frac{I}{I_{\max }}\right)^{\beta-1}
$$

where $I_{\max }$ is the maximum intensity during a certain interval. The two shape indexes $\alpha$ and $\beta$ can be evaluated by the mean value and the variance of illumination intensity.

Many studies have showed that the active output of photovoltaic generators could be described as [30]:

$$
P_{v}=A \cdot \eta \cdot I
$$

where $A$ is photoelectric array area and $\eta$ is the photoelectric transformation efficiency. Combining Equations (3) and (4), the PDF of photovoltaic generators' output power $P_{v}$ can be acquired:

$$
f\left(P_{v}\right)=\frac{\Gamma(\alpha+\beta)}{A \cdot \eta \cdot \Gamma(\alpha) \Gamma(\beta)} \cdot\left(\frac{P_{v}}{P_{v}^{\max }}\right)^{\alpha-1} \cdot\left(1-\frac{P_{v}}{P_{v}^{\max }}\right)^{\beta-1}
$$

where $P_{v}^{\max }$ is the maximum value of $P_{v}$, which can be calculated by $P_{v}^{\max }=A \cdot \eta \cdot I_{\max }$. According to (5), $P_{v}$ also follows beta distribution with shape indexes $\alpha$ and $\beta$.

\section{Risk Assessment Indices and Method}

\subsection{Risk Assessment Indices}

It should be noted that risk indices such as EENS, PLC, EFLC, and SI in [13-15] are more considered with the penetration of DGs. Therefore, in this paper, these four risk indices were used to reflect the system risk level of distribution systems. The calculation methods of these four risk indices are illustrated by the following [13]:

(1) EENS (unit: MWh/y) represents the energy that is not supplied with the penetration of DGs in the distribution system, which can be computed by (6):

$$
\mathrm{EENS}=\sum_{i=1}^{N_{L}}\left(\sum_{s \in Q_{i}} p_{T}(s) \cdot C_{0}(s)\right) \cdot T_{i}
$$

where $i$ is the load level, $N_{L}$ is the set for load levels, $T_{i}$ is the duration of $i$, and $Q_{i}$ is the set for system state $s$ at load level $i . p_{T}(s)$ and $C_{0}(s)$ are, respectively, the occurrence probability and total load curtailment of system state $s$. 
(2) PLC can be calculated by:

$$
\mathrm{PLC}=\sum_{i=1}^{N_{L}}\left(\sum_{s \in Q_{i}} p_{T}(s)\right) \cdot \frac{T_{i}}{T}
$$

(3) EFLC (unit: times/y) can be calculated by:

$$
\mathrm{EFLC}=\sum_{i=1}^{N_{L}} \sum_{s \in Q_{i}}\left(p_{T}(s) \sum_{j=1}^{m(s)} \theta_{j}\right) \cdot \frac{T_{i}}{T}
$$

where $T$ is total duration of $T_{i}$ and $m(s)$ is the set of component $j$ at system state $s$. For component $j, \theta_{j}$ represents transfer rate of component $j$, which is equal to repair rate $\mu_{j}$ in this paper.

(4) SI (unit: $\mathrm{min} / \mathrm{y}$ ) represents the equivalent duration under an entire system outage during peak conditions, which can be calculated:

$$
\mathrm{SI}=\frac{\mathrm{EENS} \times 60}{L}
$$

where $L$ is the value of peak load in 1 year.

In these four risk indices, SI is closely related to EENS. In order to calculate the index of EENS, the total load curtailment at state $s C_{0}(s)$ should be calculated beforehand; this can be calculated by optimal power flow (OPF), introduced in Section 3.3. For all of these four risk indices, the occurrence probability of system state $s p_{T}(s)$ is needed. Therefore, an enumeration method is applied for system state selection, which is introduced in Section 4.2.

\subsection{Improved Point Estimate Methods}

Point estimate method (PEM), which was firstly proposed by Hong in 1998, focuses on the statistical information that is provided by the first few central moments of the input random variables [25]. For each variable, $K$ points are used, and $K$ is a parameter named concentrations and depends on the Hong's method. These points and the function-which relates input and output variables-are used to obtain the information about the uncertainty associated with problem output random variables.

This paper relies on a $2 \mathrm{~m}$ and $2 \mathrm{~m}+1$ type scheme of PEM for computing the four risk indices described in Section 3.1, which gives a good trade-off between the solution accuracy and the computational efforts. $m$ denotes the number of random variables, which are wind speed $v$ or illumination intensity $I$. The $2 \mathrm{~m}$ type scheme of PEM uses the first two concentrations for each input random variable (i.e., $K=2$ ). For the $2 m+1$ type scheme of $P E M, K=3$.

Generally speaking, PEM is used to calculate the estimation value ( $\left.Z^{j}\right)$ of $F(X)\left(Z=F(X)=F\left(X_{1}, X_{2}\right.\right.$, $\left.\ldots, X_{m}\right)$ ) when the stochastic numerical characteristics of variables $X_{1}, X_{2}, \ldots, X_{m}$ are known $[26,27]$. The point estimation principle can be depicted as:

$$
\mathbb{E}\left(Z^{j}\right) \approx \sum_{l=1}^{m} \sum_{k=1}^{K} \omega_{l, k} F^{j}\left(\mu_{X 1}, \cdots, x_{l, k}, \cdots, \mu_{X m}\right)
$$

where $j$ is the power of $F(X), m$ is the number of DGs, $\mu_{X i}$ and $\xi_{i, k}$ are, respectively, the expectation and standard location of variable $X_{i}$, which represents illumination intensity $I$ or wind speed $v$ in this paper. $\omega_{l, k}$ represents the weight of $X_{i}$ at $x_{l, k}$, which can be calculated by (11):

$$
x_{i, k}=\mu_{X_{i}}+\xi_{i, k} \sigma_{X_{i}}, i=1,2, \cdots, m ; k=1,2, \cdots, 2 K-1
$$

where $\sigma_{X i}$ represents the variance of $X_{i}$. Standard location $\xi_{i, k}$ and weight $\omega_{l, k}$ could be determined by (12): 


$$
\left\{\begin{array}{c}
\sum_{k=1}^{K} \omega_{i, k} \cdot \xi_{i, k}^{j}=\lambda_{i, j}, \quad j=1, \cdots, 2 K-1 \\
\lambda_{i, j}=E\left[\left(x_{i}-\mu_{X_{i}}\right)^{j}\right] / \sigma_{X_{i}}{ }^{j} \\
\sum_{k=1}^{K} \omega_{i, k}=\alpha_{i}
\end{array}\right.
$$

In traditional PEM, $\sum_{k=1}^{K} \omega_{i, k}$ is set as $\frac{1}{m}$, which means the impacts of $X_{i}$ on $Z$ are the same. This assumption can simplify the calculation but may cause a big computational error as there are different impacts of $X_{i}$ on $Z$. In the improved PEM, $\sum_{k=1}^{K} \omega_{i, k}$ is set as $\alpha_{i}$, which can be determined by the analytic hierarchy process (AHP) described in [31] or the contribution of $X_{i}$ on $Z$. This equation appears to be more reasonable, as it considers the different impacts of $X_{i}$ on $Z$.

When $K=2$ and $\xi_{i, 3}=0$, the equation of (12) can be solved:

$$
\left\{\begin{array}{c}
\xi_{i, 1}=\frac{\lambda_{i, 3}}{2}+\sqrt{\frac{1}{\alpha_{i}}+\left(\frac{\lambda_{i, 3}}{2}\right)^{2}}, \xi_{i, 2}=\frac{\lambda_{i, 3}}{2}-\sqrt{\frac{1}{\alpha_{i}}+\left(\frac{\lambda_{i, 3}}{2}\right)^{2}} \\
\omega_{i, 1}=-\frac{\alpha_{i} \cdot \xi_{i, 2}}{\xi_{i, 1}-\xi_{i, 2}}, \omega_{i, 2}=\frac{\alpha_{i} \cdot \xi_{i, 1}}{\xi_{i, 1}-\xi_{i, 2}}
\end{array}\right.
$$

The calculation results based on (13) is named $2 \mathrm{~m}$ improved PEM. When $K=3$ and $\xi_{i, 3}=0,2 \mathrm{~m}+1$ improved PEM can be constructed, and the solving results of Equation (12) are:

$$
\left\{\begin{array}{c}
\xi_{i, 1}=\frac{\lambda_{i, 3}}{2}+\sqrt{\lambda_{i, 4}-\frac{3}{4} \lambda_{i, 3}{ }^{2}}, \xi_{i, 2}=\frac{\lambda_{i, 3}}{2}-\sqrt{\lambda_{i, 4}-\frac{3}{4} \lambda_{i, 3}{ }^{2}} \\
\omega_{i, 1}=\frac{1}{\xi_{i, 1}\left(\xi_{i, 1}-\xi_{i, 2}\right)}, \omega_{i, 2}=-\frac{1}{\xi_{i, 2}\left(\xi_{i, 1}-\xi_{i, 2}\right)} \\
\omega_{i, 3}=\alpha_{i}-\frac{1}{\lambda_{i, 4}-\lambda_{i, 3}{ }^{2}}
\end{array}\right.
$$

The AHP is a rough estimation of weights and may be complex in operation. Therefore, the contribution of $X_{i}$ on $Z$ which can be approximated by the capacity proportion of DG $i$ was used to calculate the value of $\alpha_{i}$.

According to Equations (10)-(14), the $j$-th raw moment of the output random vector $Z$ can be acquired. Then, the mean and the standard deviation of $Z$ can be estimated by Equation (15):

$$
\mu_{Z}=\mathbb{E}(Z) \approx \sum_{l=1}^{m} \sum_{k=1}^{K} \omega_{l, k} F\left(\mu_{X 1}, \cdots, x_{l, k}, \cdots, \mu_{X m}\right), \sigma_{Z}=\sqrt{E\left(Z^{2}\right)-\mu_{Z}^{2}}
$$

For each risk assessment index, the mean and the standard deviation can be respectively acquired by Equation (15). For a variable with a certain mathematical distribution, the mean is the most probable value of the variable [26]. Therefore, the mean of $Z, \mu_{Z}$, is used as the estimation value of each risk index in this paper.

\subsection{Optimal Power Flow Algorithm in Distribution Systems}

In distribution systems, load curtailment and generation dispatch are extensively applied to enable the system to go from urgent state to normal state. In order to obtain the system security indices in distribution systems, an optimal power flow (OPF) algorithm was presented in this paper to assess the total load curtailment $C_{0}(s)$ in state $s$. Equations (16) and (17) were used in the optimal power flow algorithm to simulate the curtailment and dispatch work and ensure the security of distribution system [32]:

$$
\min f\left(\Delta P_{G 1}, \cdots, \Delta P_{G m}, \Delta P_{L 1}, \cdots, \Delta P_{L l}\right)=\sum_{k=1}^{m} C_{G k} \Delta P_{G k}+\sum_{h=1}^{l} C_{L h} \Delta P_{L h}
$$




$$
\begin{gathered}
\text { s.t. } \sum_{k=1}^{m} \frac{\partial I_{i j}}{\partial P_{G k}} \Delta P_{G k}+\sum_{h=1}^{l} \frac{\partial I_{i j}}{\partial P_{L h}} \Delta P_{L h} \leq I_{i j}^{\max }-I_{i j}^{0} \\
\sum_{k=1}^{m} \frac{\partial P_{s}}{\partial P_{G k}} \Delta P_{G k}+\sum_{h=1}^{l} \frac{\partial P_{s}}{\partial P_{L h}} \Delta P_{L h}=0 \\
P_{G k}^{\min }-P_{G k}^{0} \leq \Delta P_{G k} \leq P_{G k}^{\max }-P_{G k}^{0} \\
0 \leq \Delta P_{L h} \leq P_{L h}^{0} \\
1 \leq k \leq m, 1 \leq h \leq l, 1 \leq i \leq N_{b}, 1 \leq j \leq N_{b}
\end{gathered}
$$

where $l$ is the number of load buses in distribution systems. $\frac{\partial z}{\partial x}\left(z=I_{i j}, P_{s} ; x=P_{G k}, P_{L h}\right)$ are sensitivity coefficients. $N_{b}$ is the number of buses; $\Delta P_{G k}$ and $\Delta P_{L h}$ are respectively the power variations of generator $k$ and load $h ; C_{G K}$ and $C_{L h}$ are respectively the control cost of generator $k$ and load $h$; and $I_{i j}$ is the current through overload line $i j$.

According to the results of this optimal power flow algorithm in distribution systems, the total load curtailment in state $s, C_{0}(s)$, can be calculated by Equation (18):

$$
C_{0}(s)=\sum_{h=1}^{l} \Delta P_{L h}+\Delta P_{d}
$$

where $\Delta P_{d}$ is the value of load curtailment caused by direct structure change of the distribution system. In line with the values of $C_{0}(s)$ and $p_{T}(s)$, the risk assessment indices in Equations (6)-(9) can be calculated.

\section{Risk Assessment Procedure for Distribution Systems}

\subsection{Structure for Risk Assessment}

To assess the four risk indices in distribution systems, PEM was used and the uncertainties of distribution generators were sufficiently considered. The flowchart of the improved PEM-based method for risk assessment of distribution systems is shown in Figure 1.

In Step $A$, active outputs of photovoltaic generators and wind generators are calculated according to the stochastic wind speed $v$ and illumination intensity $I$. In step $B$, random operation state $s$ of the distribution system was firstly selected. Then, optimal power flow algorithm in state $s$ was applied to compute the value of load curtailment based on the improved point estimate method. Then, the four risk indices listed in Section 3.1 were computed until all the random operation states $s$ are considered.

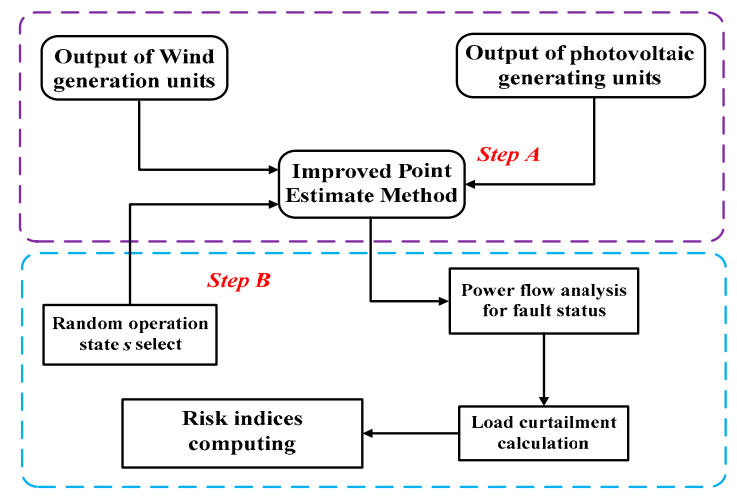

Figure 1. Diagram of the improved point estimate method (PEM)-based method for risk assessment of distribution lines in a distribution system. 


\subsection{Procedure for Calculating Risk Indices}

In [13], according to DGs' probability model, the indices of EENS, PLC, EFLC, and SI were calculated by a proposed hierarchical risk assessment method, which is the combination of a Monte Carlo simulation and an enumeration method. However, this proposed hierarchical method is relatively complex in application. In [6], $2 \mathrm{~m}+1$ PEM was utilized to assess the overload risk of a transmission line. This $2 m+1$ PEM has lower accuracy compared to a Monte Carlo simulation. However, the computational burden of $2 \mathrm{~m}+1$ PEM is greatly reduced. Consequently, the improved PEM is applied to compute the four risk indices in this paper. For comparison, the calculation results of the four risk indices based on the hierarchical risk assessment method in [13] were used as the exact values, and the error in accuracy of the improved PEM-based method for risk assessment was analyzed.

For convenience, symbol $G$ is used to represent any parameter of the four risk indices including EENS, PLC, EFLC, and SI. Namely, to calculate the parameter $G$ is to calculate these four risk indices. The designed procedure for computing the symbol $G$ based on PEM is summarized as Figure 2.

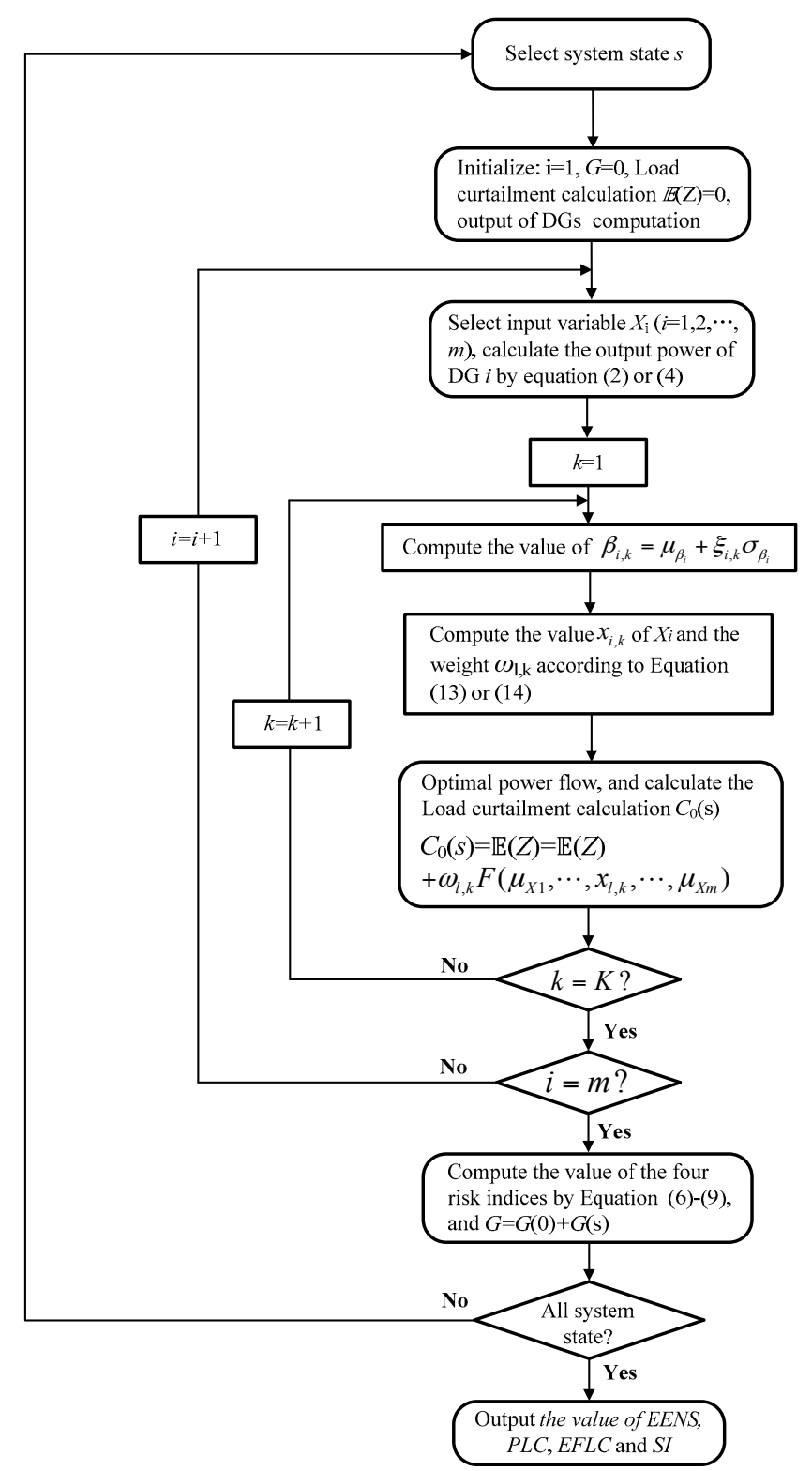

Figure 2. Flowchart of computing procedure for risk indices. 
In Figure 2, 2m and $2 \mathrm{~m}+1$ improved-PEM were used, respectively. The output power of DG $i$ is calculated by Equation (2) or (4). Further, OPF was applied to calculate the value of $C_{0}(s)$, which can be referred from Equations (16) and (17). The detailed procedure of the improved PEM-based method for computing risk indices $G$ is summarized as follows.

Step (1): Enumeration method is used to list the states of the distribution system. The occurrence probability $p_{T}(s)$ in system state $s$ can be calculated by Equation (19):

$$
p_{T}(s)=\prod_{i=1}^{N_{f}} \frac{\lambda_{i}}{\lambda_{i}+\mu_{i}} \prod_{i j=1}^{N_{n}}\left(1-\frac{\lambda_{j}}{\lambda_{j}+\mu_{j}}\right)
$$

where $N_{f}$ is the total number of failure components, $N_{n}$ is the total number of normal components. For component $i, \mu_{i}$ is the repair rate, $\lambda_{i}$ is the outage rate, and these two parameters can be obtained by statistics.

Step (2): Initialize and generate the constructed points of wind speed $v$ as well as illumination intensity $I$. Initialize the initial value of load curtailment calculation $\mathbb{E}(Z)=0$ and the initial value of $G=0$.

Step (3): Construct the parameters of improved point estimate method, which contains the following substeps:

1) Select the input variable $X_{i}(i=1,2, \ldots, m)$. For wind generating unit $i, X_{i}$ represents the stochastic wind speed $v$; for photovoltaic generating units $i, X_{i}$ represents the stochastic illumination intensity $I . m$ is the total number of wind generators and photovoltaic generators. Then, the output power of DG $i$ is calculated by Equation (2) or (4).

2) With the first central moments, standard locations $\xi_{i, k}$ and the weights $\omega_{l, k}$ for random variables are computed by Equation (12) or (13). Then, the concentrations $x_{i, k}$ can be calculated by Equation (10). Consequently, the point $\left(\mu_{x 1}, \mu_{x 2}, \ldots, x_{i, k}, \ldots, \mu_{x m}\right)$ is constructed.

3) Based on the output of DG $i$, optimal power flow in state $s$ is applied to compute the value of $C_{0}(s)$ according to Equations (15) and (16).

4) Repeat sub-steps $2-3$ until variable $k$ reaches $K$. For $2 \mathrm{~m}$ improved PEM, $K=2$; for $2 \mathrm{~m}+1$ improved PEM, $K=3$.

5) Repeat sub-steps $2-4$ until variable $i$ reaches the total number $m$, and the calculation of $G$ in state $s$ is completed.

Step (4): Repeat Steps (1)-(3) until all the states in distribution system are considered.

Step (5): Output the values of the four risk indices EENS, PLC, EFLC, and SI.

\section{Case Studies}

In order to verify the rationality of the proposed PEM-based method for risk assessment of a distribution system, IEEE 30-bus system with DGs is applied, as shown in Figure 3. For all nodes, the upper voltage bound is 1.06 p.u. and lower voltage bound is 0.94 p.u. in this paper [6]. All the cases are achieved by MATLAB in an Advanced Micro Devices 64 Dual Core $3.3 \mathrm{GHz}$ PC. For photovoltaic farms, shape indexes $\alpha=15.42, \beta=4.3, A=2.17 \mathrm{~m}, \eta=13.53 \%$. For wind farms, rated wind speed $v_{N}=15 \mathrm{~m} / \mathrm{s}$, cut-in wind speed $v_{\text {in }}=4 \mathrm{~m} / \mathrm{s}$, cut-out wind speed $v_{\text {out }}=20 \mathrm{~m} / \mathrm{s}$, shape index $k=6.23$, and scale index $c=10.43$. 


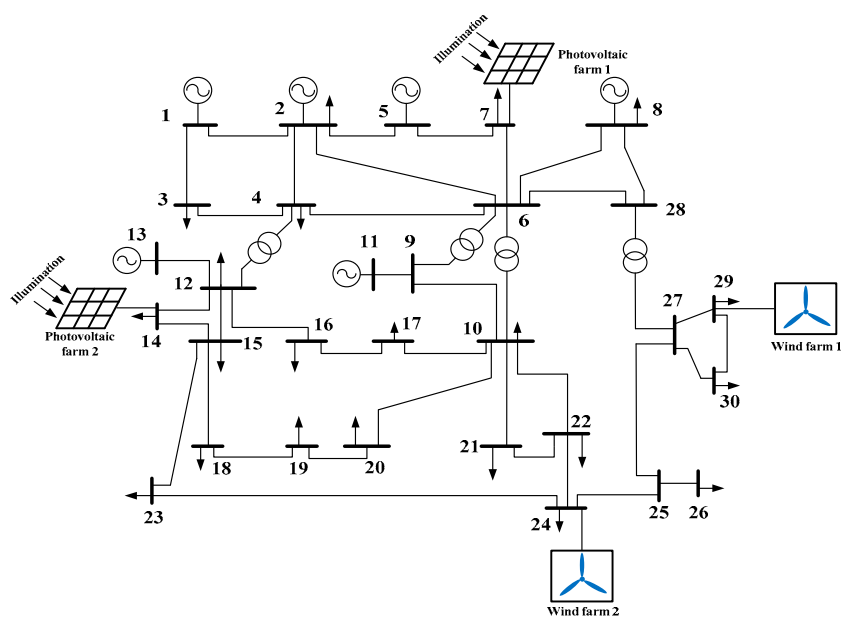

Figure 3. Modified IEEE 30-bus system with distributed generators (DGs).

\subsection{Calculation of Risk Indices Based on PEM}

In this modified IEEE 30-bus system, two wind farms and two photovoltaic farms are connected to nodes $7,14,24$, and 29 . The capacity proportion of these four farms is $20 \%: 30 \%: 20 \%: 30 \%$. However, the ratio of DGs' total generation capacity to the total load $K_{\mathrm{d}}$ varies from 0 to 1 . With the known capacity proportion of DGs, the value of $\alpha_{i}$ can be obtained: $\alpha_{1}=0.2, \alpha_{2}=0.3, \alpha_{3}=0.2, \alpha_{4}=0.3$.

According to the designed procedure for computing risk indices in Figure 2 and the parameters of DGs, the calculation results of EENS, PLC, EFLC, and SI, based on $2 \mathrm{~m}$ and $2 \mathrm{~m}+1$ improved PEM, are listed in Tables 1 and 2, respectively.

Table 1. Calculation results of risk indices based on $2 \mathrm{~m}$ improved PEM.

\begin{tabular}{ccccc}
\hline \multirow{2}{*}{$K_{\mathbf{d}}$} & \multicolumn{4}{c}{ 2m PEM } \\
\cline { 2 - 5 } & EENS (MWh/y) & PLC & EFLC (Times/y) & SI (min/y) \\
\hline 0 & 3151.2 & $62,247 \times 10^{-7}$ & 2.4899 & 66.716 \\
0.2 & 2382.5 & $59,981 \times 10^{-7}$ & 2.3992 & 50.441 \\
0.4 & 1945.7 & $53,176 \times 10^{-7}$ & 2.1285 & 41.193 \\
0.6 & 1744.4 & $48,635 \times 10^{-7}$ & 1.9454 & 36.932 \\
0.8 & 1619.1 & $47,516 \times 10^{-7}$ & 1.9006 & 34.279 \\
1 & 1580.3 & $45,243 \times 10^{-7}$ & 1.8097 & 33.457 \\
\hline
\end{tabular}

$K_{\mathrm{d}}$ : generation capacity; EENS: expected energy not supplied; PLC: probability of load curtailment; EFLC: expected frequency of load curtailment; SI: severity index.

Table 2. Calculation results of risk indices based on $2 \mathrm{~m}+1$ improved PEM.

\begin{tabular}{ccccc}
\hline \multirow{2}{*}{$K_{\mathbf{d}}$} & \multicolumn{4}{c}{ 2m + 1 PEM } \\
\cline { 2 - 5 } & EENS (MWh/y) & PLC & EFLC (Times/y) & SI (min/y) \\
\hline 0 & 3156.7 & $62,293 \times 10^{-7}$ & 2.4917 & 66.832 \\
0.2 & 2385.4 & $60,033 \times 10^{-7}$ & 2.4013 & 50.502 \\
0.4 & 1948.2 & $53,212 \times 10^{-7}$ & 2.1285 & 41.246 \\
0.6 & 1747.3 & $48,687 \times 10^{-7}$ & 1.9475 & 36.993 \\
0.8 & 1621.8 & $47,551 \times 10^{-7}$ & 1.9020 & 34.336 \\
1 & 1582.5 & $45,285 \times 10^{-7}$ & 1.8114 & 33.504 \\
\hline
\end{tabular}

As can be seen from Tables 1 and 2, with the increase of total generation capacity $K_{d}$, all of these risk indices decrease gradually. When the generation capacity $K_{d}=0$, which means that DGs are not permeated, the value of EENS is about 3156.7 MWh/year. However, EENS decreases by nearly a half 
and drops to about $1582.5 \mathrm{MWh}$ /year when the generation capacity $K_{\mathrm{d}}$ rises to 1 . Analogously, the value of PLC decreases from about 0.0062293 to 0.0045285 when generation capacity $K_{d}$ rises from 0 to 1.

The variation tendency of SI is consistent with the variation tendency of EENS since the index of SI is calculated by EENS, which is shown in Section 3.1. Similarly, the variation tendency of EFLC is consistent with the variation tendency of PLC. In addition, the calculation results of risk indices based on $2 \mathrm{~m}$ improved PEM are very close with the calculation results of risk indices based on $2 \mathrm{~m}+1$ improved PEM.

From Tables 1 and 2, EENS and SI decrease smoothly with the increment of generation capacity $K_{\mathrm{d}}$. In addition, the slope of EENS and SI decreases with the growth of generation capacity $K_{d}$. Therefore, the decreasing trend of EENS and SI is not as tangible as before, decreasingly when generation capacity $K_{\mathrm{d}}$ increases.

As shown in Tables 1 and 2, the decrease of EENS, PLC, ELIC, and SI slows when generation capacity $K_{\mathrm{d}}$ increases to about 0.6 . Namely, a large value of $K_{\mathrm{d}}$ that promotes the decrease of risk indices of distribution systems is not significantly remarkable. However, the consumption of wind and photovoltaic energy becomes an increasing problem with the increasing generation capacity $K_{\mathrm{d}}$. It is with reluctance that too much wind and photovoltaic power is abandoned in distribution systems. Therefore, it is not necessary to increase the value of $K_{\mathrm{d}}$ to a certain large degree.

\subsection{Deviation and Computational Cost Comparison}

In order to illustrate the effectiveness of this improved PEM-based method for risk assessment in distribution systems, the calculation results of EENS, PLC, EFLC, and SI are shown in Table 3.

Table 3. Calculation results of risk indices based on hierarchical risk assessment method.

\begin{tabular}{ccccc}
\hline \multirow{2}{*}{$K_{\mathbf{d}}$} & \multicolumn{4}{c}{ Hierarchical Method } \\
\cline { 2 - 5 } & EENS (MWh/y) & PLC & EFLC (Times/y) & SI (min/y) \\
\hline 0 & 3178.5 & $62,434 \times 10^{-7}$ & 2.4974 & 67.293 \\
0.2 & 2406.6 & $60,164 \times 10^{-7}$ & 2.4066 & 50.95 \\
0.4 & 1963.8 & $53,353 \times 10^{-7}$ & 2.1341 & 41.577 \\
0.6 & 1759.5 & $48,812 \times 10^{-7}$ & 1.9525 & 37.251 \\
0.8 & 1634.6 & $47,677 \times 10^{-7}$ & 1.9071 & 34.608 \\
1 & 1589.2 & $45,407 \times 10^{-7}$ & 1.8163 & 33.646 \\
\hline
\end{tabular}

Comparing the calculation results of risk indices in Tables 1 and 2 with Table 3, it can be seen that they are very close in value. However, the calculation results of these improved PEM-based methods are all less than that of the hierarchical risk assessment method since PEM is an order approximation, which ignores the higher-order terms. Besides, the calculation results of $2 m+1$ improved PEM are closer to the exact value than the calculation results of $2 \mathrm{~m}$ improved PEM, as $2 \mathrm{~m}$ improved PEM is a third-order approximation but $2 \mathrm{~m}+1$ improved PEM is a fourth-order approximation, in fact.

According to the analysis in Section 3.1, the variation tendency of SI is consistent with the variation tendency of EENS, and the variation tendency of EFLC is consistent with the variation tendency of PLC. Therefore, the deviation comparison of EENS and PLC are only needed to be analyzed, which are shown in Figures 4 and 5.

In Figures 4 and 5 , errors $\delta_{1}$ and $\delta_{2}$ are respectively calculated by comparing the results of EENS and PLC in Tables 1 and 2 with Table 3. As can be seen from Figures 4 and 5, the maximum value of computational error $\delta_{1}$ is about $0.99 \%$ and the maximum value of computational error $\delta_{2}$ is about $0.37 \%$. The computational errors $\delta_{1}$ and $\delta_{2}$ are all less than $1 \%$, which greatly verifies the effectiveness of this improved PEM-based method for risk assessment of distribution systems. Also, the computational error $\delta_{2}$ of PLC is less than the computational error $\delta_{1}$ of EENS. This is because optimal power flow algorithm (based on Equations (15) and (16)) is used in the calculation procedure for EENS. 
Compared with hierarchical risk assessment method-which is a combination of the Monte Carlo method and enumeration method-in fact, the computational burden of this PEM-based method is greatly reduced. When generation capacity $K_{\mathrm{d}}=0.5$, the computational costs of risk indices are as listed in Table 4.

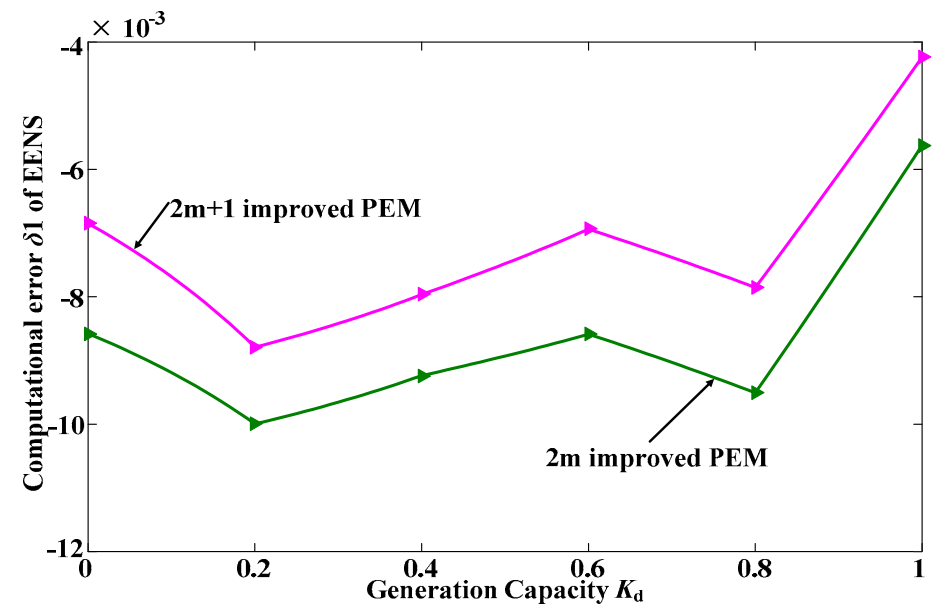

Figure 4. The computational error $\delta_{1}$ of EENS in pace with $K_{\mathrm{d}}$.

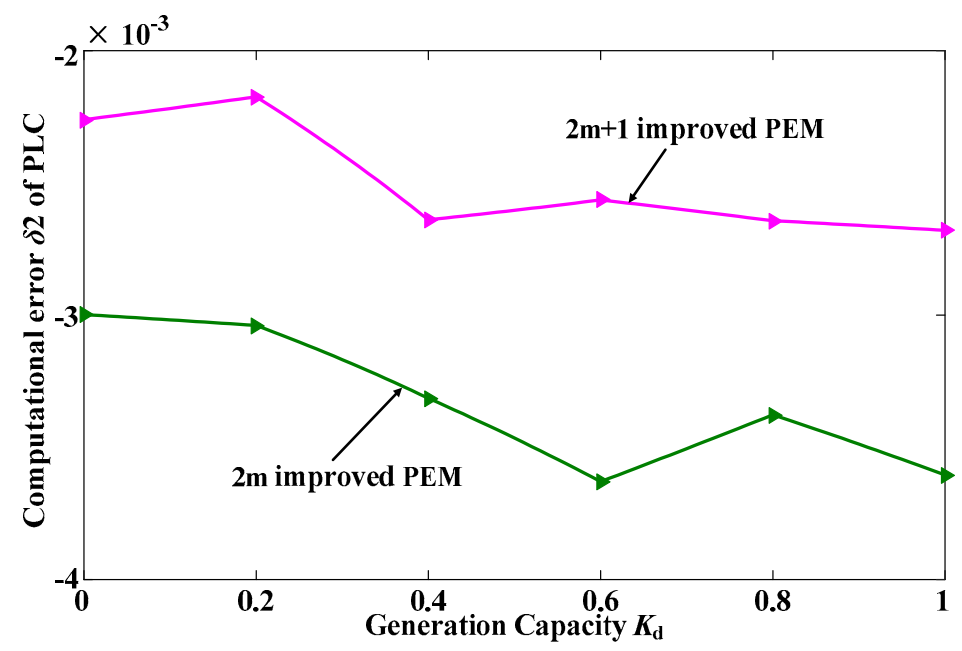

Figure 5. The computational error $\delta_{2}$ of PLC in pace with $K_{\mathrm{d}}$.

Table 4. Calculation results of risk indices based on hierarchical risk assessment method.

\begin{tabular}{cccc}
\hline \multirow{2}{*}{ Risk Indices } & \multicolumn{3}{c}{ The Computational Costs of Risk Indices(s) } \\
\cline { 2 - 4 } & 2m Improved PEM & 2m + 1 Improved PEM & Hierarchical Method \\
\hline EENS & 25.845 & 27.742 & 67.293 \\
PLC & 1.985 & 2.031 & 5.950 \\
EFLC & 2.157 & 2.192 & 6.107 \\
SI & 25.845 & 27.742 & 67.293 \\
\hline
\end{tabular}

As can be seen from Table 4, the computational costs of this improved PEM-based method for risk assessment in distribution systems are much less than the computational costs of the hierarchical method. For EENS, the computational costs of $2 \mathrm{~m}$ improved PEM and $2 \mathrm{~m}+1$ improved PEM are 25.845 $\mathrm{s}$ and $27.742 \mathrm{~s}$, respectively, but the computational cost of the hierarchical method is $67.293 \mathrm{~s}$. This is 
because the Monte Carlo method, with many computational hurdles, is used in the hierarchical method. On the contrary, only a few numerical operations are needed in the improved PEM. The calculation of risk indices has to be evaluated only $K$ times for each input variable, which is stochastic wind speed $v$ or stochastic illumination intensity $I$ at the $K$ points.

In addition, the computational costs of $2 \mathrm{~m}+1$ improved PEM-based method are almost the same compared to $2 \mathrm{~m}$ improved PEM-based method, as the only difference between these two methods are a few numerical operations. These simulations have demonstrated that the improved PEM-based method has high accuracy and highly reduced computational costs, therefore, it is extremely applicable for risk assessment of distribution systems.

\subsection{Influence of DGs on System Risk}

Many researches have shown that many other aspects such as type, location, dispersion, and capacity proportion of DGs have great influences on the system risk indices. In this part, the influences of these aspects are analyzed.

In Section 5.1, two wind farms and two photovoltaic farms are connected to nodes 7, 14, 24, and 29. Suppose that four wind generating units are respectively connected to nodes $7,14,24$, and 29 and their capacity proportion is still 20\%:30\%:20\%:30\%. This case is named case 1 in this paper. According to the developed $2 m+1$ improved PEM-based method for risk assessment in Figure 2, the four system risk indices of EENS, PLC, EFLC, and SI can be acquired when generation capacity $K_{d}=0.2$, which can be seen in Table 5 .

Table 5. Calculation results of risk indices in case 1.

\begin{tabular}{lllll}
\hline \multirow{2}{*}{$K_{\mathrm{d}}$} & \multicolumn{4}{l}{$\mathbf{2 m}+\mathbf{1}$ Improved PEM } \\
\cline { 2 - 5 } & EENS (MWh/y) & PLC & EFLC (Times/y) & SI (min/y) \\
\hline 0.2 & 2131.7 & $56,823 \times 10^{-7}$ & 2.2729 & 45.131 \\
\hline
\end{tabular}

As can be seen from Table 5, the index of EENS decreases from 2385.4 MWh/y to $2131.7 \mathrm{MWh} / \mathrm{y}$ and the values of other indices are also cut down distinctly. Therefore, for DGs' type, wind farms' power support is better than that of photovoltaic farms. These results reflect that the randomness of the output power of photovoltaic farms is even bigger than the active output power of wind farms.

To illustrate the influence of DGs' location, suppose that two wind farms and two photovoltaic farms are respectively connected to nodes $3,14,19$, and 30 in this part, and their capacity proportion is still 20\%:30\%:20\%:30\%. This case is named case 2 in this paper. Likewise, the calculation results of system risk indices based on $2 \mathrm{~m}+1$ improved PEM, when generation capacity $K_{\mathrm{d}}=0.2$, are outlined in Table 6.

Table 6. Calculation results of risk indices in case 2.

\begin{tabular}{lllll}
\hline \multirow{2}{*}{$K_{\mathrm{d}}$} & \multicolumn{4}{l}{ 2m + 1 Improved PEM } \\
\cline { 2 - 5 } & EENS (MWh/y) & PLC & EFLC (Times/y) & SI (min/y) \\
\hline 0.2 & 2193.6 & $57,232 \times 10^{-7}$ & 2.2893 & 46.442 \\
\hline
\end{tabular}

Comparing the calculation results of Table 6 with Table 2, it is observed that the index of EENS decreases from 2385.4 MWh/y to 2193.6 MWh/y and the values of other indices are also cut down distinctly. This comparison shows that the locations of nodes $3,14,19$, and 30 are more suitable for optimal siting of DGs than nodes 7, 14, 24, and 29. However, these locations may be not the most optimal siting for DGs, and how to ascertain the optimal siting of DGs is worth further study.

To illustrate the influence of DG dispersion, suppose that three wind farms and three photovoltaic farms are respectively connected to nodes $3,7,14,24,29$, and 30 in this part, and their capacity 
proportion is 15\%:15\%:20\%:15\%:15\%:20\%. This case is named case 3 in this paper and the calculation results of system risk indices are outlined in Table 7.

Table 7. Calculation results of risk indices in case 3.

\begin{tabular}{lllll}
\hline \multirow{2}{*}{$K_{\mathrm{d}}$} & \multicolumn{2}{l}{ 2m + 1 Improved PEM } & \\
\cline { 2 - 5 } & EENS $(\mathbf{M W h} / \mathbf{y})$ & PLC & EFLC (Times/y) & SI (min/y) \\
\hline 0.2 & 2058.4 & $55,785 \times 10^{-7}$ & 2.2314 & 43.579 \\
\hline
\end{tabular}

In case 3, the total proportion of wind farms and photovoltaic farms is still $50 \%: 50 \%$ but the dispersion degree of DGs in case 3 is enhanced. The results in Tables 2 and 7 show that system risk indices decrease along with dispersion degree increment. Therefore, when installed, DGs should be as highly dispersed as possible for the optimal siting of DGs for application.

The influence of DGs' capacity proportion reflects the influence of DGs' type on system risk indices. As wind farms' power support is better than that of photovoltaic farms, the values of system risk indices are cut down when the wind generating units' capacity proportion is increased.

These test results illustrate that appropriate DGs' siting and sizing have great influences on the system risk indices. For the sizing of DGs, system risk indices increase along with the DG's capacity, however, the corresponding investment costs will increase. For the appropriate siting of DGs, different locations may suit different types of DGs, and DGs' dispersion can decrease the value of system risk indices.

\section{Conclusions}

DGs connected to a distribution system could cause some critical risks to distribution systems from security and economy aspects. In order to reasonably assess the risks of distribution systems with penetrating DGs, four risk indices-EENS, PLC, EFLC, and SI-were used in this paper to reflect the system risk level in distribution systems. The output uncertainties of DGs were depicted by some certain mathematical distributions. Then, an improved PEM-based method was proposed to calculate these four system risk indices. In this improved PEM-based method, enumeration method was used to list the states of distribution systems, and an improved PEM was presented to deal with the uncertainties of DGs.

Test results of case studies have shown that this proposed PEM-based method is highly effective for assessing the risk of a distribution system with DGs, as it has a high computational accuracy and highly reduced computational costs compared with other risk assessment methods. Simulation results also demonstrate that total generation capacity, type, location, dispersion, and capacity proportion of DGs have great influences on the system risk indices. The determining method of appropriate DGs' siting and sizing, considering the operation risk of a distribution system with DGs, is worthy of further research.

Acknowledgments: This work was supported by The National Key Technology R\&D Program of China (2013BAA02B01) and Qinghai Province Key Laboratory of Photovoltaic Grid Connected Power Generation Technology (2014-Z-Y34A).

Author Contributions: Qingwu Gong proposed the concrete ideas of the proposed optimization method. Jiazhi Lei performed the simulations and wrote the manuscript. Hui Qiao and Jingjing Qiu gave some useful suggestions to the manuscript. Both of the authors revised the manuscript.

Conflicts of Interest: The authors declare no conflict of interest.

\section{References}

1. Arabali, A.; Ghofrani, M.; Etezadi-Amoli, M. Stochastic Performance Assessment and Sizing for a Hybrid Power System of Solar/Wind/Energy Storage. IEEE Trans. Sustain. Energy 2014, 5, 363-371. [CrossRef] 
2. Zhao, H.; Guo, S. External Benefit Evaluation of Renewable Energy Power in China for Sustainability. Sustainability 2015, 7, 4783-4805. [CrossRef]

3. Wu, Q.; Peng, C. Comprehensive Benefit Evaluation of the Power Distribution Network Planning Project Based on Improved IAHP and Multi-Level Extension Assessment Method. Sustainability 2016, 8, 796. [CrossRef]

4. Al Kaabi, S.S.; Zeineldin, H.H.; Khadkikar, V. Planning Active Distribution Networks Considering Multi-DG Configurations. IEEE Trans. Power Syst. 2014, 29, 785-793. [CrossRef]

5. Negnevitsky, M.; Nguyen, D.H.; Piekutowski, M. Risk Assessment for Power System Operation Planning with High Wind Power Penetration. IEEE Trans. Power Syst. 2015, 30, 1359-1368. [CrossRef]

6. Li, X.; Zhang, X.; Wu, L. Transmission Line Overload Risk Assessment for Power Systems with Wind and Load-Power Generation Correlation. IEEE Trans. Smart Grid 2015, 3, 1233-1242. [CrossRef]

7. Marsadek, M.; Mohamed, A.; Mohd, Z. Assessment and classification of line overload risk in power systems considering different types of severity functions. WSEAS Transcr. Power Syst. 2010, 3, 182-191.

8. Feng, Y.Q.; Wu, W.C.; Zhang, B.; Li, W.Y. Power system operation risk assessment using credibility theory. IEEE Trans. Power Syst. 2008, 23, 1309-1318. [CrossRef]

9. Ding, Y.; Wang, P. Reliability and price risk assessment of a restructured power system with hybrid market structure. IEEE Trans. Power Syst. 2006, 21, 108-116. [CrossRef]

10. Celli, G.; Ghiani, E.; Pilo, F.; Soma, G.G. Active distribution network reliability assessment with a pseudo sequential Monte Carlo method. In Proceedings of the IEEE PowerTech, Trondheim, Norway, 19-23 June 2011; pp. 1-8.

11. Atwa, Y.M.; El-Saadany, E.F. Reliability evaluation for distribution system with renewable distributed generation during islanded mode of operation. IEEE Trans. Power Syst. 2009, 24, 572-581. [CrossRef]

12. Li, W.; Zhou, J.; Lu, J.; Yan, W. Incorporating a combined fuzzyand probabilistic load model in power system reliability assessment. IEEE Trans. Power Syst. 2007, 22, 1386-1388. [CrossRef]

13. Jia, H.; Qi, W.; Liu, Z.; Wang, B. Hierarchical Risk Assessment of Transmission System Considering the Influence of Active Distribution Network. IEEE Trans. Power Syst. 2015, 2, 1084-1093. [CrossRef]

14. Li, W.; Zhou, J.; Xie, K.; Xiong, X. Power system risk assessment using a hybrid method of fuzzy set and Monte Carlo simulation. IEEE Trans. Power Syst. 2008, 23, 336-343.

15. Billinton, R.; Wang, P. Reliability cost/worth assessment ofdistribution systems incorporating time-varying weather conditions andrestorations resources. IEEE Trans. Power Deliv. 2002, 17, 260-265.

16. Soroudi, A.; Amraee, T. Decision making under uncertainty in energy systems: State of the art. Renew. Sustain. Energy Rev. 2013, 28, 376-384. [CrossRef]

17. Soroudi, A.; Ehsan, M. IGDT based robust decision making tool for DNOs in load procurement under severe uncertainty. IEEE Trans. Smart Grid 2013, 4, 886-895. [CrossRef]

18. Bertsimas, D.; Litvinov, E.; Sun, X.A.; Zhao, J.; Zheng, T. Adaptive robust optimization for the security constrained unit commitment problem. IEEE Trans. Power Syst. 2013, 28, 52-63. [CrossRef]

19. Baudrit, C.; Dubois, D.; Guyonnet, D. Joint propagation and exploitation of probabilistic and possibilistic information in risk assessment. IEEE Trans. Fuzzy Syst. 2006, 14, 593-608. [CrossRef]

20. Liu, K.Y.; Sheng, W.; Hu, L.; Liu, Y. Simplified probabilistic voltage stability evaluation considering variable renewable distributed generation in distribution systems. IET Gener. Transm. Distrib. 2015, 9, 1464-1473. [CrossRef]

21. Liu, Z.; Wen, F.; Ledwich, G. Optimal siting and sizing of distributed generators in distribution systems considering uncertainties. IEEE Trans. Power Deliv. 2011, 26, 2541-2551. [CrossRef]

22. Rei, A.M.; Schilling, M.T. Reliability assessment of the Brazilianpower system using enumeration and Monte Carlo. IEEE Trans. Power Syst. 2008, 23, 1480-1487. [CrossRef]

23. Martinez-Velasco, J.A.; Guerra, G. Parallel Monte Carlo approach for distribution reliability assessment. IET Gener. Transm. Distrib. 2014, 8, 1810-1819. [CrossRef]

24. Conejo, A.J.; Carrión, M.; Morales, J.M. Decision Making under Uncertainty in Electricity Markets; Springer: New York, NY, USA, 2010; Volume 1.

25. Hong, H.P. An efficient point estimate method for probabilistic analysis. Reliab. Eng. Syst. Saf. 1998, 59, 261-267. [CrossRef]

26. Morales, J.M.; Perez-Ruiz, J. Point estimate schemes to solve the probabilistic power flow. IEEE Trans. Power Syst. 2007, 4, 1594-1601. [CrossRef] 
27. Su, C.L. Probabilistic load-flow computation using point estimate method. IEEE Trans. Power Syst. 2005, 20, 1843-1851. [CrossRef]

28. Papavasiliou, A.; Oren, S.S.; O'Neill, R.P. Reserve requirements for wind power integration: A scenario-based stochastic programming framework. IEEE Trans. Power Syst. 2011, 26, 2197-2206. [CrossRef]

29. Li, J.; Wei, W.; Xiang, J. A simple sizing algorithm for stand-alone PV/wind/battery hybrid microgrids. Energies 2012, 5, 5307-5323. [CrossRef]

30. Kratochvil, J.A.; Boyson, W.E.; King, D.L. Photovoltaic Array Performance Model; Technical Report; Sandia National Laboratories: New Mexico, NM, USA, 2004.

31. Saaty, T.L. Analytic hierarchy process. In Encyclopedia of Biostatistics; John Wiley and Sons, Inc.: Somerset, NJ, USA, 2001; pp. 19-28.

32. Stott, B.; Marinho, J.L. Linear programming for power-system network security applications. IEEE Trans. Power Appl. Syst. 1979, 3, 837-848. [CrossRef]

(C) 2017 by the authors. Licensee MDPI, Basel, Switzerland. This article is an open access article distributed under the terms and conditions of the Creative Commons Attribution (CC BY) license (http://creativecommons.org/licenses/by/4.0/). 